\title{
Recién nacido pretérmino con quilotórax congénito bilateral, una entidad infrecuente que amenaza la vida
}

\author{
Luz A. Aguilar-López* \\ Laura L. Rodríguez-Moncada** \\ Ángela M. Diaz-Diaz *** \\ Juan S. Peinado-Acevedo ${ }^{* * * *}$
}

\begin{abstract}
* Médica General. Instituto del Corazón de Bucaramanga. Facultad de Ciencias de la Salud. Universidad Autónoma de Bucaramanga. Bucaramanga. Santander. Colombia.

** Médica General. Clínica FOSCAL. Facultad de Ciencias de la Salud. Universidad Autónoma de Bucaramanga. Bucaramanga. Santander. Colombia. *** Médica especialista en Pediatría. Gerencia Servicios de Salud y Gestión de Empresas. Coordinadora Médica UCIP y Pediatría. Clínica FOSCAL. Docente Programa de Medicina. Universidad Autónoma de Bucaramanga. Bucaramanga. Santander. Colombia.

**** Médico Interno. XII Nivel de Medicina. Facultad de Ciencias de la Salud. Universidad Autónoma de Bucaramanga. Bucaramanga. Santander. Colombia.

Correspondencia: Dra. Luz Andrea Aguilar López. Direccion: Carrera 26 № 35 - 170 - Altos de cañaveral V etapa. Bloque 9. Apto 302. Floridablanca. Santander. Colombia. Teléfono (+57) 6381330 - (301) 3368430. Correo electrónico: I.andrea87@hotmail.com
\end{abstract}

\section{RESUMEN}

El quilotórax corresponde a la acumulación de linfa en el espacio pleural. El diagnóstico se confirma por la presencia de quilomicrones en el líquido pleural, sin embargo en la práctica clínica se utilizan los criterios de Büttiker, para establecer el diagnóstico. El objetivo de esta publicación es presentar el caso de un recién nacido con quilotórax congénito bilateral, realizando una revisión actualizada del tema, que incentive la recolección de datos y la generación de guías nacionales para su abordaje estandarizado. Se trató de un recién nacido pretérmino, quien tras parto por cesárea presentó síndrome de distrés respiratorio, ameritó terapia temprana de surfactante logrando retiro de ventilación mecánica al día de vida, con posterior presentación de signos de dificultad respiratoria y hallazgo radiológico y ecográfico de derrame pleural bilateral. Se realizó toracentesis con citoquímico compatible con quilotórax. Nuevamente ameritó soporte ventilatorio invasivo, toracostomía cerrada bilateral y nutrición parenteral, con posterior evolución clínica satisfactoria. MÉD.UIS. 2017;30(1):87-92.

Palabras claves: Quilotórax Congénito. Síndrome de Dificultad Respiratoria del Recién Nacido. Derrame pleural. Prematuro.

\section{Preterm newborn with bilateral congenital chylothorax, a rare life-threatening entity} ABSTRACT

Chylothorax refers to the accumulation of lymphatic fluid in the pleural space. Diagnosis is confirmed by the presence of chylomicrons in the pleural fluid, however in clinical practice Büttiker criteria are widely used. The aim of this publication is to present the case of a newborn with bilateral congenital chylothorax with an updated review of the literature that encourages data collection and generation of national guidelines for standardized approach. This was a newborn preterm, who after a cesarean delivery presented respiratory distress syndrome who required early surfactant therapy achieving withdrawal of mechanical ventilation on his first day, with subsequent presentation of signs of respiratory distress, and a radiological and ultrasound finding of bilateral pleural effusion. Thoracentesis was performed obtaining cytochemical compatible with chylothorax. Again required invasive ventilatory support, closed bilateral thoracostomy and parenteral nutrition, with a satisfactory clinical course. MÉD.UIS. 2017;30(1):87-92.

Keywords: Congenital Chylothorax. Respiratory Distress Syndrome, Newborn. Pleural Effusion. Premature. 
¿Cómo citar este artículo?: Aguilar-López LA, Rodríguez-Moncada LL, Diaz-Diaz AM, PeinadoAcevedo JS. Recién nacido pretérmino con quilotórax congénito bilateral, una entidad infrecuente que amenaza la vida. MÉD.UIS. 2017;30(1):87-92.

\section{INTRODUCCIÓN}

El quilotórax se define como la acumulación de linfa en la cavidad pleural. Fue descrito por primera vez por Pusek en $1917^{1}$. Como patología neonatal es un hallazgo poco común; su epidemiología nacional y local es deconocida, sin embargo, estudios internacionales realizados en Israel y Alemania, muestran una incidencia entre $1: 7300^{2}$ a 1:24 $000^{3}$ recién nacidos vivos respectivamente, siendo más frecuente en el género masculino, con una proporción $2: 1^{2,3,4,5}$ y la mortalidad perinatal oscila entre el 15 y el $30 \%$.

El conducto torácico representa el canal más importante en el desarrollo del quilotórax, este se origina de partir de la cisterna de quilo cerca a la línea media a nivel de la segunda vértebra lumbar y pasa a través del hiato diafragmático aórtico hacia el hemitórax derecho por la región medial, para finalmente desembocar en el sistema venoso a través del ángulo venoso ${ }^{7,8}$. Existen dos grandes grupos etiológicos, el quilotórax congénito y el adquirido. El congénito esta dado por causas idiopáticas, siendo fetos y neonatos los grupos etáreos más relevantes ${ }^{4,9,10,1,112}$. El de origen adquirido, es secundario a complicaciones de cirugías cardiotorácicas o hace parte de un síndrome clínico específico (Ver Tabla 1).

El diagnóstico se puede sospechar de forma prenatal, con el hallazgo de derrame pleural en el feto, mediante ecografía obstétrica ${ }^{4,13}$ y se confirma en el periodo postnatal mediante el hallazgo de quilomicrones en el líquido pleural o según los criterios Büttiker en el análisis citoquímico de líquido pleural, con la presencia de triglicéridos $>100 \mathrm{mg} / \mathrm{dl}(>1,1 \mathrm{mmol} / \mathrm{l})$, con $>1000$ células/ $\mu$ l y un predominio de linfocitos ( $>$ $80 \%)^{6}$. La electroforesis de proteínas, se considera el gold standard ${ }^{14,15,16}$, sin embargo es poco utilizado por su baja disponibilidad. El curso clínico generalmente es tórpido y las complicaciones más frecuentes son secundarias a la pérdida de quilo: hipovolemia, hipoalbuminemia, hipogammaglobulinemia e hipofibrinogenemia y trastornos hidroelectrolíticos ${ }^{6}$.

El tratamiento puede ser médico o quirúrgico y se basa en aliviar la afectación respiratoria, mantener un estado nutricional adecuado, intentar disminuir la producción de quilo y disminuir la mortalidad asociada ${ }^{16}$.

El objetivo de este artículo es presentar un caso clínico de un recién nacido pretérmino con quilotórax bilateral congénito y hacer una revisión actualizada sobre su abordaje diagnóstico y terapéutico a nivel respiratorio y nutricional que incentive la investigación en el tema y la publicación de casos. Lo anterior es especialmente importante para llenar los vacíos del conocimiento existentes, por ejemplo, la ausencia de datos sobre incidencia local, para poder desarrollar ensayos clínicos que generen datos basados en la evidencia para el desarrollo de guías de manejo.

\section{DESCRIPCIÓN DE CASO}

Recién nacido pretermino 33 semanas, con peso de $1400 \mathrm{gr}$, producto de parto por cesárea, por actividad uterina relacionada con ruptura prematura de membranas de tres días de evolución, sin datos de corioamnionitis materna; quien desarrollo síndrome de dificultad respiratoria agudo moderado, dado por aleteo nasal constante, quejido audible, tiraje subcostal acentuado con Silverman de seis al nacer y desaturación persistente. Se manejó inicialmente con aporte de oxígeno por cámara cefálica al 50\%, con posterior requerimiento de dispositivo mecánico nasal de presión positiva, sin mejoría ameritando soporte ventilatorio invasivo, esquema de surfactante pulmonar orotraqueal a dosis de 1,6 mg cada seis horas por dos dosis, con evolución clínica favorable que permitió extubación a las primeras 24 horas.

Al segundo día de vida presentó nuevamente aleteo nasal, desincronización de los movimientos respiratorios toraco-abdominales y tirajes universales con desaturación progresiva y sostenida asociada; requirió nuevamente ventilación invasiva y se inició aporte nutricional por sonda orogástrica. Se realizó radiografía simple de tórax con evidencia de borramiento de ángulos costofrenicos bilateral e imagen de vidrio esmerilado de predominio izquierdo, ecografía de bases pulmonares con derrame pleural bilateral, líquido anecógeno, sin 
ecos ni septos, $30 \mathrm{cc}$ izquierdo y 25 cc derecho (Ver Imagen 1), ecocardiograma trastorácico con hipertensión pulmonar secundaria a ductus arterioso persistente 2,1 $\mathrm{mm}$ con cortocircuito de izquierda a derecha, trastorno de la coagulación, tiempo parcial de tromboplastina de 78,3 segundos, hipoalbuminemia (albumina 2,7g/dl) e hipernatremia (sodio: $167 \mathrm{mEq} / \mathrm{L}$ ), así como acidemia persistente con pH en 7,21 $\mathrm{pCO}_{2} 42 \mathrm{mmHg}$ y $\mathrm{pO}_{2}$ de $117 \mathrm{mmHg}$.

Posteriormente se realizó toracostomía cerrada bilateral, con salida de líquido turbio color amarillo, se procesó estudio de citoquímico (Ver Tablas 2-3), gram, cultivo y antibiograma siendo negativos para infección. Se interpretó reporte de citoquímico como quilotórax congénito, ameritando cambio a nutrición parenteral total con lípidos de cadena mediana ajustados en cantidad según evolución. En las siguientes 48 horas presentó clínica favorable, logrando realizar extubación orotraqueal al octavo día de vida. Se realizó retiro de toracostomía cerrada derecha el día 11 e izquierda el día 12 de vida, permaneció con soporte nutricional parenteral hasta el día 15, pasando a soporte enteral con aumentos progresivos acordes a su tolerancia. Traslado a sala de hospitalización general luego de 24 días de estancia en Unidad de Cuidados Intensivos. Se le dio de alta hospitalaria a los 48 días de vida, sin oxígeno suplementario, alimentación por succión, y adecuada recuperación nutricional peso de egreso 2585 .

Tabla 1. Causas de quilotórax adquirido ${ }^{11,12,26,27,28,29}$

\begin{tabular}{|c|c|c|c|c|c|c|}
\hline latrogenia & $\begin{array}{c}\text { Origen torácico } \\
\text { no cardiaco }\end{array}$ & $\begin{array}{c}\text { Origen torácico } \\
\text { cardiaco }\end{array}$ & Neoplasias & Genéticas & $\begin{array}{c}\text { Anormalidades } \\
\text { pulmonares y linfáticas }\end{array}$ & Infecciosas \\
\hline $\begin{array}{l}\text {-Por cirugía cardiaca } \\
\text { congénita } \\
\text {-Por cirugía de hernia } \\
\text { diafragmática } \\
\text {-Por cateterismo de la } \\
\text { vena subclavia } \\
\text {-Por cirugía de anillo } \\
\text { vascular }\end{array}$ & $\begin{array}{l}\text {-Hernias diafragmáticas } \\
\text {-Agenesia bilateral de la } \\
\text { vena cava superior con } \\
\text { obstrucción del conducto } \\
\text { torácico }\end{array}$ & $\begin{array}{l}\text {-Cardiopatías } \\
\text { congénitas }\end{array}$ & $\begin{array}{l}\text {-Tumores pleurales } \\
\text {-Linfangiohemangioma } \\
\text { mediastínico. }\end{array}$ & $\begin{array}{l}\text {-Síndrome Down: Agresividad } \\
\text { en caso de trisomía } \\
21 \text { robertsoniana (46,XY,t(14q21q) } \\
\text { (q10;q10) b21): Diagnostico } \\
\text { concomitante de displasia linfática } \\
\text { generalizada } \\
\text {-Síndrome Noonan } \\
\text {-Síndrome Turner } \\
\text {-Síndrome de Gorham-Stout } \\
\text {-Miopatía miotubular asociada a X } \\
\text {-Mutación missense en } \\
\text { integrina a9b1 } \\
\text {-Hydrops fetal }\end{array}$ & $\begin{array}{l}\text {-Linfangiectasia pulmonar } \\
\text { congénita primaria } \\
\text {-Higroma quístico } \\
\text {-Fistula traqueo esofágica tipo H } \\
\text {-Secuestro pulmonar } \\
\text {-Corioangioma placentario }\end{array}$ & $\begin{array}{l}\text {-Torch } \\
\text {-Neumonia }\end{array}$ \\
\hline
\end{tabular}

Fuente: Autores

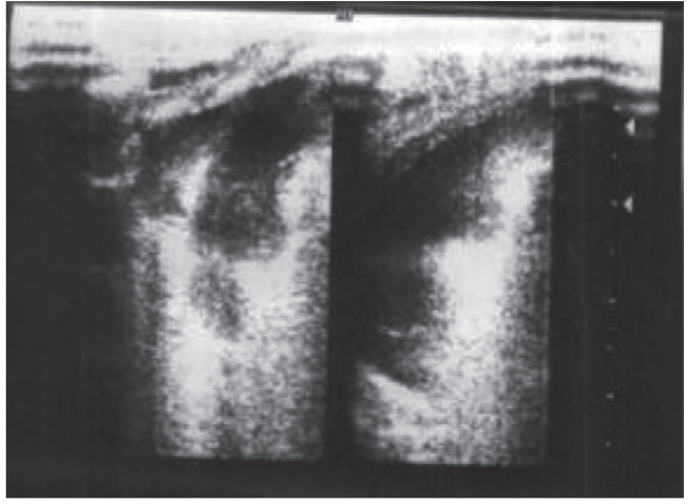

Imagen 1. Ecografía de bases pulmonares: Líquido anecógeno sin ecos ni septos $30 \mathrm{cc}$ izquierdo y $25 \mathrm{cc}$ derecho.

Fuente: Archivo Radiologos especializados, Foscal

\section{Discusión}

El síndrome de dificultad respiratoria del recién nacido, es una entidad con múltiples etiologías, tales como: taquipnea transitoria del recién nacido, síndrome de aspiración meconial, síndrome de dificultad respiratoria e hipertensión pulmonar persistente del recién nacido; otras como el derrame pleural son menos frecuentes, más aún, si es secundario a la presencia de quilotórax ${ }^{2,5,9,13}$, etiología identificada en el abordaje de una transición fallida o tardía de la circulación fetal a la neonatal, como ocurrió en nuestro caso, donde el diagnóstico inicial fue síndrome de dificultad respiratoria de etiología no clara pero que posteriormente ante los hallazgos imagenológicos se consideró el diagnóstico de quilotórax. En los niños en edad escolar, la prevalencia de derrame pleural es mayor, principalmente debido a que la patología infecciosa es una causa frecuente, así mismo, el quilotórax en este grupo etario también se puede presentar generalmente debido a otras causas como complicación de cirugía cardiotorácica o por malignidad ${ }^{10,17}$. 
Tabla 2. Citoquímico de Líquido pleural

\begin{tabular}{|c|c|}
\hline DERECHO & IZQUIERDO \\
\hline \multicolumn{2}{|c|}{ Examen físico sin centrifugar } \\
\hline Color: Amarillo & Color: Amarillo \\
\hline Olor: Sui Generis & Olor: Sui Generis \\
\hline $\begin{array}{l}\text { Aspecto: Turbio } \\
\text { Aspecto: Turbio }\end{array}$ & $\begin{array}{l}\text { Aspecto: Turbio } \\
\text { Aspecto: Turbio }\end{array}$ \\
\hline PH: 7 & $\mathrm{PH}: 7$ \\
\hline $\begin{array}{l}\text { Coagulo de fibrina: } \\
\text { Negativo }\end{array}$ & $\begin{array}{l}\text { Coagulo de fibrina: } \\
\text { Negativo }\end{array}$ \\
\hline \multicolumn{2}{|c|}{ Examen físico líquido centrifugado } \\
\hline Color: Amarillo & Color: Amarillo \\
\hline Aspecto: Turbio & Aspecto: Turbio \\
\hline \multicolumn{2}{|c|}{ Microscópico } \\
\hline Hematíes: 250/mm3 & Hematíes: 213/mm3 \\
\hline Hematíes normales: $20 \%$ & $\begin{array}{l}\text { Hematíes normales: } \\
20 \%\end{array}$ \\
\hline Hematíes crenados: $80 \%$ & $\begin{array}{l}\text { Hematíes crenados: } \\
80 \%\end{array}$ \\
\hline Leucocitos: 4/mm3 & Leucocitos: 2/mm3 \\
\hline Linfocitos: $100 \%$ & Linfocitos: $100 \%$ \\
\hline
\end{tabular}

Fuente: autores

Tabla 3. Citoquímico liquido pleural

\begin{tabular}{|l|l|}
\hline Triglicéridos: $850 \mathrm{mg} / \mathrm{dl}$ & Triglicéridos: $915 \mathrm{mg} / \mathrm{dl}$ \\
\hline Glucosa: $295 \mathrm{mg} / \mathrm{dl}$ & Glucosa: $501 \mathrm{mg} / \mathrm{dl}$ \\
\hline Proteínas: $200 \mathrm{mg} / \mathrm{dl}$ & Proteínas: $100 \mathrm{mg} / \mathrm{dl}$ \\
\hline $\mathrm{LDH}: 43 \mathrm{U} / \mathrm{L}$ & $\mathrm{LDH}: 35 \mathrm{U} / \mathrm{L}$ \\
\hline
\end{tabular}

Fuente: Autores

La presentación bilateral suele ser la más usual ${ }^{2,5,18}$, como lo ocurrido en el paciente; en caso de compromiso de un solo hemitórax, el derecho suele ser el más frecuentemente afectado, probablemente debido a la ubicación del conducto torácico ${ }^{2,4,18}$. La clínica depende del momento en el cual se desarrolle el quilotórax; en la etapa fetal por ejemplo, puede resultar en hipoplasia pulmonar y presentar manifestaciones tempranas más graves, mientras que en etapa neonatal, se generan signos de dificultad respiratoria como taquipnea, retracciones y cianosis de intensidad leve a severa, sintomatología que presentó el paciente. Los hallazgos físicos, aunque de menor valor en el neonato que en los individuos de edad avanzada, incluyen disminución de los ruidos respiratorios y sonido mate a la percusión, estos hallazgos no fueron evidenciados en el paciente, por lo que el diagnóstico fue basado principalmente en los hallazgos imagenológicos ${ }^{9,12}$.

Así mismo, la herramienta diagnóstica también estará determinada según el momento en que se genere la patología, en la etapa prenatal, el método diagnóstico fue sugerido inicialmente por Defoort y Thiery en 1978, quienes usaron el ultrasonido en modo B para visualizar el polihidramnios y posteriormente la ecografía en escala de grises para visualizar líquido en la cavidad pleural4,13,19. Ahora bien, el pilar diagnóstico en la etapa postnatal lo constituye la presencia de quilomicrones en el líquido pleural ${ }^{14,15,20}$, sin embargo dada la poca disponibilidad de la electroforesis de proteínas en los centros de atención neonatal, cobran mayor importancia los criterios descritos por Büttiker.

A la fecha, dado que se conoce que el líquido pleural de los fetos con quilotórax tiene niveles mayores de IgG, se considera que otra herramienta diagnóstica podría corresponder a la presencia de una relación IgG en el líquido pleural/sérico elevada ${ }^{21}$. El aspecto lechoso en el estudio macroscópico, puede sugerir la presencia de quilotórax, sin embargo, no se considera patognomónico debido a que se correlaciona con el contenido de lípidos en el derrame, el cual dependerá del estado nutricional que tiene el neonato al momento de desarrollar el cuadro clínico ${ }^{22}$. De ahí que, Staats et al, concluyeran en su estudio sobre el perfil de lipoproteínas presente en los derrames pleurales, quiloso vs no quiloso, que es un mal indicador de su origen, dándole especial importancia a los valores de triglicéridos, pues estos últimos sí se podrían acercar a la determinación de efusión quilosa. Se encontró entonces que valores de triglicéridos superiores a $110 \mathrm{mg} / \mathrm{dL}$ son muy sugestivos de una efusión quilosa, mientras que los valores de triglicéridos entre 50 y $110 \mathrm{mg} / \mathrm{dL}$ requieren análisis de electroforesis de lipoproteínas para confirmar el diagnóstico ${ }^{14}$. Es raro poder confirmar el diagnóstico de quilotórax, debido a la escasa disponibilidad de la electroforesis de lipoproteínas, 
aun en instituciones de alta complejidad, como en la que fue atendido el paciente. El citoquímico de este caso no es el típicamente descrito en los casos de quilotórax, (ausencia de leucocitos mayor de 1000 células/mm3), sin embargo, hay que recordar que todos los criterios diagnósticos en medicina tienen una sensibilidad y especificidad que no son del $100 \%$, es decir, hay pacientes que sin cumplir los criterios diagnósticos pueden tener la enfermedad, en este caso, el aspecto macroscópico, la evolución clínica favorable al instaurar el manejo y la ausencia de otras enfermedades probables, fue fundamental para realizar el diagnóstico. Adicionalmente, la madre de este paciente ingresó a la institución, remitida desde su lugar de residencia, luego de identificar trabajo de parto prematuro inminente, con controles prenatales inadecuados, dadas las condiciones de acceso al servicio de salud en su lugar de residencia, por lo cual no fue posible una realizar el diagnóstico prenatal.

La etiología del quilotórax en este caso se interpretó como idiopática debido a que el recién nacido no tenía otros signos clínicos que sugirieran cromosomopatía o anormalidades genéticas mayores, se cree que en estos casos podrían existir anormalidades genéticas menores que conducen a un retraso en el desarrollo normal de los linfáticos en el periodo neonatal, el cual mejora rápidamente, evitando nuevos episodios de quilotórax en la vida adulta, esto se sustenta con el mayor riesgo que tienen los hijos de madres que ya han tenido otros hijos con quilotórax ${ }^{23}$.

En cuanto al tratamiento del quilotórax, aún no se han estandarizado guías de manejo por lo que se realiza un tratamiento guiado por objetivos, los cuales se basan en aliviar la afectación respiratoria, mantener un estado nutricional adecuado y disminuir tanto la producción de quilo, como la morbilidad asociada a la enfermedad. Se han descrito diferentes estrategias de manejo según el momento del diagnóstico.

En la etapa posnatal, el manejo puede ser clasificado en tres categorías: Manejo de la enfermedad subyacente, tratamiento conservador y tratamiento quirúrgico ${ }^{24}$. No es objeto de esta revisión, sin embargo se debe enfocar el proceso diagnóstico diferencial para así, direccionar la identificación y el manejo de la enfermedad subyacente. El tratamiento conservador inicialmente implica la sustitución de los nutrientes perdidos en el quilo y el drenaje quirúrgico de grandes quilotórax, por lo que se indica el inicio de nutrición enteral con triglicéridos de cadena media, los cuales son absorbidos directamente en el sistema porta, sin pasar por el sistema linfático intestinal, lo que reduce el flujo de quilo en el conducto torácico. Si el paciente continua con eliminación de quilo, se indica reposo intestinal e inicio de nutrición parenteral total $^{2,24}$, tratamiento realizado en el paciente. Otra de las medidas usadas como tratamiento conservador, es el uso de somatostatina, la cual se indica si no hay respuesta a las medidas dietéticas, su tasa de éxito puede ser alrededor del 50\% y se basa en su efecto atenuante sobre el flujo de la linfa del conducto torácico ${ }^{25}$. Finalmente, el manejo quirúrgico se recomienda en casos donde a pesar de tratamiento conservador, el paciente drena más de $100 \mathrm{~mL} / \mathrm{Kg}$ de peso corporal por día y en quienes se ha producido un rápido declive en el estado nutricional a pesar del tratamiento conservador ${ }^{24}$. Se incluye manejo con pleurodesis o pleurectomía, ligadura del conducto torácico y finalmente toracoscopia o toracotomía ${ }^{25}$.

\section{Conclusiones}

El quilotórax en etapa prenatal y neonatal es un reto médico pues hay muy poca información actualizada, sobre su diagnóstico y tratamiento, por lo que se debe incentivar su estudio, con la realización de ensayos clínicos que permitan generar guías prácticas clínicas, con el fin de estandarizar el diagnóstico y tratamiento de esta patología.

Por el momento, existen criterios aún vigentes descritos por Büttiker et al. para su diagnóstico y debe correlacionarse con la clínica del paciente. Por otro lado, el hallazgo prenatal, determina una mejor sobrevida a los pacientes que cursan con esta patología dado que el tratamiento in útero ha aumentado la tasa de sobrevida y menor tasa de morbilidad a largo plazo.

El pilar del tratamiento en la etapa postnatal, se basa en el diagnóstico oportuno y el manejo nutricional del paciente, usando como herramienta principal la nutrición enteral con triglicéridos de cadena media. El pronóstico se correlaciona con la presentación clínica del paciente, y con la concomitancia de otras patologías que condicionen un pobre desenlace.

\section{Referencias Bibliográficas}

1. Pisek G. Report of a chylothorax. Arch Pediatr. 1917;34:929-37

2. Ergaz Z, Bar-Oz B, Yatsiv I, Arad I. Congenital chylothorax: Clinical course and prognostic significance. Pediatr Pulmonol. 2009;44(8):806-11. 
3. Bialkowski A, Poets CF, Franz AR. Congenital chylothorax: a prospective nationwide epidemiological study in Germany. Arch Dis Child - Fetal Neonatal Ed [Internet]. 2015;100(2):F16972. Available from: http://fn.bmj.com/lookup/doi/10.1136/ archdischild-2014-307274.

4. Rocha G. Pleural effusions in the neonate. Curr Opin Pulm Med 2007;13:305-11.

5. Lee C-J, Tsao P-N, Chen C-Y, Hsieh W-S, Liou J, Chou H-C. Prenatal Therapy Improves the Survival of Premature Infants with Congenital Chylothorax. Pediatr Neonatol [Internet]. Elsevier Taiwan LLC; 2016;57(2):127-32. Available from: http:// linkinghub.elsevier.com/retrieve/pii/S1875957215001138.

6. Büttiker V, Fanconi S, Burger R. Chylothorax in children guidelines for diagnosis and management.Anionic Chest. 1999 Sep;116(3):682-7.

7. Schoenwolf, Bleyl, Brauer, Francis-West. Larsen's Human Embryology, 5th Edition. Churchill Livingstone; December 24, 2014.

8. Keith L. Moore, T. V. N. Persaud, Mark G. Torchia. The Developing Human: Clinically Oriented Embryology, 10th. Saunders; April 20, 2015.

9. Shih YT, Su PH, Chen JY, Lee IC, Hu JM, Chang HP. Common etiologies of neonatal pleural effusion. Pediatr Neonatol [Internet]. Elsevier Taiwan LLC; 2011;52(5):251-5. Available from: http://dx.doi.org/10.1016/j.pedneo.2011.06.002.

10. Chernick V, Reed MH. Pneumothorax and chylothorax in the neonatal period. J Pediatr. 1970;76:624-32. [PubMed].

11. Downie L, Sasi A, Malhotra A. Congenital chylothorax: associations and neonatal outcomes. J Paediatr Child Health 2014;50:234-8.

12. Tutor JD. Chylothorax in infants and children. Pediatrics. 2014 Apr;133(4):722-33. doi: 10.1542/peds.2013-2072.

13. Greenough A, Roberton NRC. Acute respiratory disease in the newborn. In: Rennie JM, Roberton NRC, editors. Textbook of neonatology, 3rd ed. London: Churchill Livingstone; 1999. pp. 481-607.

14. Staats BA, Ellefson RD, Budahn LL, Dines DE, Prakash UB, Offord $\mathrm{K}$. The lipoprotein profile of chylous and nonchylous pleura effusions. Mayo Clin Proc. 1980 Nov;55(11):700-4. Avaible from http://www.ncbi.nlm.nih.gov/pubmed/7442324.

15. Seriff NS, Cohen ML, Samuel P, Schulster PL. Chylothorax: diagnosis by lipoprotein electrophoresis of serum and pleura fluid. Thorax. 1977;32(1):98-100.

16. Benítez I, Copons C, Castillo F. Tratamiento del quilotórax. An
Pediatría Contin [Internet]. 2008;6(3):159-65. Available from: http://www.apcontinuada.com/es/tratamiento-delquilotorax/ articulo/80000329/

17. Chan S, Lau W, Wong W, et al. Chylothorax in children after congenital heart surgery. Ann Thorac Surg. 2006;82:1650-7.

18. Van Straaten HL, Gerards LJ, Krediet TG. Chylothorax in the neonatal period. Eur J Pediatr 1993;152:2 - 5

19. Defoort P, Thiery M. Antenatal diagnosis of congenital chylothorax by gray scale sonography. J Clin Ultrasound 1978;6:47 - 48 .

20. Hillerdal G. Chylothorax and pseudochylothorax. Eur Respir J. 1997;10(5):1157-62.

21. Tsukimori K, Nakanami N, Fukushima K,Yoshimura T, Hikino S, Nakano H. Pleural fluid/serum immunoglobulin ratio is a diagnostic marker for congenital chylothorax in utero. J Perinat Med 2006;34:313-317.

22. Maldonado F, Hawkins FJ, Daniels CE, Doerr CH, Decker PA, Ryu JH. Pleural Fluid Characteristics of Chylothorax. Mayo Clinic Proceedings. 2009;84(2):129-133.

23. Chang YL, Lien R, Wang CJ, Chang SD, Soong YK. Congenital chylothorax in three siblings. Am J Obstet Gynecol. 2005 Jun;192(6):2065.-6

24. Fox GF, Challis D, O'Brien KK, Kelly EN, Ryan G. Congenital chylothorax in siblings. Acta Paediatr. 1998;87(9):1010-2.

25. McGrath EE, Blades Z, Anderson PB. Chylothorax: Aetiology, diagnosis and therapeutic options. Respir Med [Internet]. Elsevier Ltd; 2010;104(1):1-8.

26. Cannizzaro V, Frey B, Bernet-Buettiker V. The role of somatostatin in the treatment of persistent chylothorax in children. Eur Cardio-thoracic Surg. 2006;30(1):49-53.

27. Ochiai M, Hikino S, Nakayama H, Ohga S, Taguchi T, Hara T. Nonimmune hydrops fetalis due to generalized lymphatic dysplasia in an infant with Robertsonian trisomy 21. Am J Perinatol 2006;23:63-6.

28. ChenCP. Fetal therapy and cytogenetic testing: prenatal detection of chromosome aberration during thoracocentesis for congenital chylothorax by karyotyping from pleural effusion fluid and review of the literature. Genet Couns 2005;16:301-5.

29. Gleason CA, Devaskar SU. Avery's diseases of the newborn. 9th ed. Philadelphia: Saunders; 2012. p. 682-4.

30. Chiang MC. Congenital Chylothorax: Antenatal Intervention, Survival, and Outcome. Pediatr Neonatol [Internet]. Elsevier Taiwan LLC; 2016;57(2):85-6. Available from: http://dx.doi. org/10.1016/j.pedneo.2016.01.003 